\title{
Identidad social: conductas sanitarias y solidarias en México ante el confinamiento por COVID-19*14
}

Melissa García Meraz

Doctora en Psicología Social Universidad Nacional Autónoma de México UNAM Correo electrónico: melissaunam@yahoo.com.mx
Recibido: 03/08/2020

Evaluado: 09/11/2020 Aceptado: 03/03/2021

\section{Resumen}

En concordancia con la teorización y hallazgos empíricos del Modelo Elaborado de la Identidad Social, se adaptó una escala para evaluar las conductas solidarias durante la emergencia derivada de la enfermedad COVID-19. 600 mujeres y 400 hombres respondieron la batería en redes sociales y otros contactos digitales. Los resultados del Análisis Factorial Confirmatorio mostraron un ajuste adecuado del instrumento $\chi 2(247)=732.53 \mathrm{p}<.001, \mathrm{CFI}=.96$; RMSEA $=.045 ; \mathrm{SRMR}=.049$. Un segundo modelo, que incluyó la exposición a la emergencia sanitaria, probó diversas hipótesis relacionadas con la predicción de la ejecución de conductas solidarias, los resultados mostraron un ajuste adecuado $\chi 2(247)=732.53 \mathrm{p}<.001, \mathrm{CFI}=.96 ; \mathrm{RMSEA}=.045 ; \mathrm{SRMR}=.045$. La identidad social, derivada de un destino común ante la emergencia, y la observación de conductas solidarias predicen el seguimiento de normas sanitarias, brindar apoyo socioemocional y coordinado en redes.

Palabras clave

Identidad, solidaridad, COVID-19, normas, México.

14 Para citar este artículo: García-Meraz, M. (2022). Identidad social: conductas sanitarias y solidarias en México ante el confinamiento por COVID-19. Informes Psicológicos, 22(1), pp. 235-250. http://dx.doi.org/10.18566/infpsic.v22n1a14

* Trabajo financiado por el proyecto UNAM-PAPIIT IA311120. 


\title{
Social identity: health and solidarity behaviors in Mexico in the face of confinement by COVID-19
}

\begin{abstract}
In line with the theorization and empirical findings of the Elaborated Model of Social Identity, a scale was adapted to evaluate solidarity behaviors during the emergency derived from the COVID-19 disease. 600 women and 400 men answered the survey on social networks and other digital contacts. The results of the Confirmatory Factor Analysis showed an adequate adjustment of the instrument $\chi 2(247)=732.53 p<.001$, $\mathrm{CFI}=.96 ; \mathrm{RMSEA}=.045 ; \mathrm{SRMR}=.049$. A second model, which included exposure to the health emergency, tested various hypotheses related to the prediction of the execution of solidarity behaviors. The results showed an adequate adjustment $\chi 2$ (247) $=732.53 p<.001$, CFI $=.96$; RMSEA $=.045$; SRMR $=.045$. The social identity derived from a common destiny in the face of the emergency along with the observation of solidary behaviors predict the follow-up of sanitary norms and provide socio-emotional and coordinated support in networks.
\end{abstract}

Keywords Identity, solidarity, COVID-19, norms, Mexico.

\section{Identidade social: comportamentos de saúde e solidariedade no México diante do confinamento por COVID-19}

Resumo

De acordo com a teorização e achados empíricos do Modelo Elaborado de Identidade Social, foi adaptada uma escala para avaliar comportamentos de solidariedade durante a emergência decorrente da doença COVID-19. 600 mulheres e 400 homens responderam ao repositório nas redes sociais e outros contatos digitais. Os resultados da Análise Fatorial Confirmatória mostraram um ajuste adequado do instrumento $\chi 2(247)=732.53 p<.001, \mathrm{CFI}=.96$; RMSEA $=.045 ;$ SRMR $=.049$. Um segundo modelo, que incluiu a exposição à emergência sanitária, testou várias hipóteses relacionadas à predição da execução de comportamentos solidários, os resultados mostraram um ajuste adequado $\chi 2(247)=732.53 p<.001$, $\mathrm{CFI}=.96 ; \mathrm{RMSEA}=.045 ; \mathrm{SRMR}=.045$. A identidade social, derivada de um destino comum diante da emergência, e a observação de comportamentos solidários predizem o cumprimento de normas sanitárias, fornecem suporte socioemocional e articulado em redes.

Palavras chave Identidade, solidariedade, COVID-19, normas, México. 


\section{ntroducción}

En los últimos años ha habido un creciente interés sobre el comportamiento y acción de los individuos en situaciones de emergencia. De hecho, el interés se ha volcado en la investigación referente a cómo los individuos, en grupo o en colectivos que se encuentran en una situación estresante y de emergencia, tal como un sismo o un ataque terrorista, logran ayudarse entre sí, ya sea escapando o proveyendo apoyo emocional o físico hacia otros sobrevivientes (Drury, Cocking, \& Reicher, 2009b, 2009a; Reicher, 2008). Por ello, la investigación reciente se ha focalizado en mostrar que, más allá de la visualización clásica acerca del comportamiento de masas como irracionales y destructivas, los colectivos están caracterizados por una amplitud de situaciones que implican el comportamiento solidario o de apoyo (Cocking, Drury, \& Reicher, 2009; Drury et al., 2009b; Zeitz, Tan, Grief, Couns, \& Zeitz, 2009). En este sentido, cabe destacar el alto grado de cooperación entre personas y aun, podríamos remarcar, la presencia de una conducta colectiva autoorganizada y cooperativa en situaciones de emergencia (Solnit, 2009). De acuerdo con la teoría de la identidad social, Drury ha propuesto que la conducta colectiva es posible en situaciones de emergencia de masas o de desastres donde la amenaza de muerte (real o implícita) está presente (Drury, 2018, 2020; Drury, Brown, González, \& Miranda, 2016): Esta conducta proviene del hecho de compartir una identidad social, gestada por la experiencia colectiva de emergencia $y$, con ello, un sentimiento de estar juntos bajo un destino común (Drury, 2018).

En nuestro país existen una serie de situaciones de emergencia que se han estudiado bajo la óptica de las ciencias sociales. Ejemplo de ello es la existencia de conductas solidarias entre personas que experimentan un sismo o una inundación, aunado a las emergencias sanitarias. En 2012, Fernández-Poncela planteó desde la psicología colectiva el efecto de la identidad colectiva sobre el comportamiento de las masas y la difusión del rumor durante la emergencia sanitaria de la influenza provocada por el virus H1N1. Aun cuando la autora identifica la identidad social como relevante, no contrasta datos empíricos que pudiesen defender sus tesis (Fernández-Poncela, 2012). Es así que la evidencia científica y sistemática relacionada al comportamiento de los colectivos, desde una mirada psico sociocultural, es aún insuficiente.

En México, durante el mes de marzo de este año se registraron los primeros casos de COVID-19, causada por el nuevo coronavirus SARS-CoV-2 y, con el venir de los días se declaró el estado de emergencia y la necesidad del seguimiento de normas sanitarias tales como la sana distancia, el lavado de manos y quedarse en casa (Salud, 2020). Durante los primeros meses de confinamiento y restricción de actividades, las personas han reportado pérdida de empleos así como consecuencias emocionales, psicológicas y físicas (Brooks et al., 2020; Sandín, Valiente, García-Escalera, \& Chorot, 2020; Zandifar \& Badrfam, 2020), aunado al incremento en la desconfianza acerca de la veracidad de la información, hacia las normas sanitarias y las medidas gubernamentales, lo que 
ha incrementado la infodemia y la falta de seguimiento de normas sanitarias (Redacción, 2020; UNAM, 2020). Sin embargo, y a pesar de los retos planteados para conocer cómo promover las conductas solidarias, incluidas el seguimiento de normas sanitarias, se tiene nula evidencia acerca de estudios planteados desde la psicología social, particularmente, desde las tesis de la ejecución de conductas solidarias a partir de la observación y de una identidad social compartida.

De acuerdo con esto, y con el objetivo de conocer más acerca de los elementos que impactan en las conductas solidarias ya señaladas, se aplicó una escala de 25 reactivos bajo el Modelo Elaborado de la Identidad Social (MEIS) de Drury et al. (2016). Este modelo en parte, aplica la teoría del aprendizaje social, observar conductas solidarias, emocionales y sociales y predice la ejecución de ayuda en situaciones de emergencia. Sin embargo, también hipotetiza que, más allá de la observación, la identificación social, y no así la identidad individual, ante una exposición de emergencia, predice en mayor medida la ejecución de conductas solidarias. Esto es posible dado que la exposición a la emergencia posibilita una suerte de sentimiento de destino común que, a su vez, elicita en mayor medida las conductas solidarias. Dado que, en México, se ha promovido una serie de normas de cuidado ante la emergencia sanitaria (quédate en casa, sana distancia, lavado de manos) incluidas como ejecución de conductas de autocuidado y solidarias. De esta manera, el objetivo fue identificar si la identificación social ante la emergencia predice la ejecución de conductas solidarias o de seguimiento de normas sanitarias. Se tomaron reactivos de la escala aplicada por Drury et al. (2016) así como la creación de reactivos específicos para la emergencia sanitaria COVID-19.

El presente trabajo comienza con una exposición de la teoría clásica sobre el comportamiento de la masa y su respuesta en teorías más contemporáneas sobre la acción colectiva. Se destaca la contribución clásica de Le Bon (1962) y la propuesta moderna del Modelo Elaborado de la Identidad Social en su adaptación a las situaciones de emergencia. Posterior al método, se presentan los resultados en términos del Análisis Factorial Confirmatorio (AFC) de las dimensiones conceptualizadas a partir del estudio posterior al sismo en Chile de 2010 (Drury et al., 2016), así como las dimensiones creadas para dar cuenta del seguimiento de normas sanitarias para COVID-19 y conductas solidarias en redes sociales virtuales. Los resultados concluyen con la presentación del Modelo de Ecuación Estructural.

\section{Teorías psicosociales sobre el comportamiento colectivo: de la irracionalidad del colectivo a las teorías de la identidad social}

Las teorías tradicionales relacionadas con los comportamientos de masas pueden describirse desde las ideas de Le Bon. Para su época, Le Bon constituía uno de los primeros teóricos en enfrentar el tema; sin embargo, su visión no era positiva, ni libertaria. Para él, las personas que se vinculan a un colectivo o una "masa" podrían considerarse como más sensibles y con 
una ausencia colectiva de todos los pensamientos racionales relacionados con el yo, la responsabilidad y la capacidad para los pensamientos individuales. Masas crédulas, influenciables y con sentimiento de poder invencible. Como entidad no racional, la multitud está dominada por emociones, impulsos, movilidad e irritabilidad; además, los sentimientos siempre son excesivos, desbordados y, por ello, cualquier intento de negociación o pensamiento racional es imposible.

Hay varias consecuencias en la caracterización de la masa de Le Bon. Reicher (2008) ha señalado que, desde la teoría clásica, la acción de masas es imposible. Desde esta primera visualización, la masa es solo un concentrado irracional y emocional de personas sin nada que decir. La consecuencia del modelo de Le Bon es la pérdida de la persona, la irracionalidad y la responsabilidad. Esto indica que hemos anulado el proceso psicológico de los sujetos sociales. Por supuesto, esto no ha hecho sino abonar más a la división clásica acerca del individuo y la sociedad. Ahí donde impera la estructura social, el individuo no tiene nada que decir. Sin embargo, lo cierto es que, aun en los contextos de índole más colectiva o masiva, los individuos tienen un papel importante en juego. Quizás, y cuanto más intentemos anular al individuo, es más importante su papel. Si consideramos que la masa se define con base en la ausencia de individuos, entonces, ésta es la consecuencia de perder el aspecto más importante de la argumentación: la individualidad racional y única (Featherstone, 1995). Con ello, la conducta de la masa no tiene más que lamentarse por haber perdido al individuo y seguir teorizando acerca de él y su ausencia, echando de menos la ausencia de la racionalidad por haber perdido la unicidad de ser persona. Esto, por supuesto, le da tal importancia al individuo que elimina también la posibilidad de que la conducta de la masa sea vista, realmente, como una acción colectiva.

Pero, ¿es posible que el colectivo tenga algo qué decir? En estudios recientes se ha encontrado que el pertenecer a un colectivo que tiene una lucha en común empodera a los individuos (Uluğ \& Acar, 2019), incluso las personas en protestas experimentan cambios significativos en su identidad. La identidad es vista como un factor de movilización y acceso al poder político (Melucci, 1989; Sudbury, 2012). De hecho, y a pesar de que no se logren los objetivos dentro de las luchas colectivas, los individuos participantes conservan un sentimiento de empoderamiento que va más allá de la formación del colectivo y que lleva a la autonomía cultural y la identidad colectiva (Buechler, 1993). Aunado a ello, las personas que participan en huelgas desarrollan una actitud más crítica hacia aquellos en el poder y una autoconcepción más consciente de clase (Fantasia, 1989). Parece que, después de todo, los colectivos tienen mucho que decir.

Para Reicher (2008), si deseamos comprender la naturaleza de la acción colectiva, necesitaríamos un modelo teórico que conecte de nuevo a la sociedad con la identidad y a la identidad con la sociedad; esto, para el autor, solo es posible a través de una teoría de la identidad social en contraposición a la identidad individual que pueda entenderse desde el punto de vista de la psicología de las masas. Los primeros intentos de comprender mejor el comportamiento de las multitudes se basaron en los argumentos de Turner, Oakes, Haslam, \& McGarty (1994) sobre el comportamiento colectivo y la influencia social como 
una función de la autocategorización y la identidad compartidas. Desde una aproximación más novedosa del Modelo Elaborado de la Identidad Social (Elaborated Social Identity Model ESIM) sobre el comportamiento de la multitud, Reicher (2008) ha argumentado que la identidad social es una función de los procesos intergrupales. A partir de esta teoría, es posible interpretar el conflicto en función de dos grupos con identidades incompatibles, como los disturbios en los que la policía y los manifestantes se han visto como parte de diferentes grupos y comparten diferentes identidades (Turner, Oakes, Haslam, \& McGarty, 1994). Además, el cambio de autocategorización, las personas involucradas en disturbios pueden ser identificadas como opuestas a la policía (Drury \& Reicher, 1999; Reicher, 2008). De hecho, las personas dentro de los colectivos pueden cambiar su posición y transformar la forma como entienden su propia posición y acción (Reicher, 2008).

Aunado a ello, es importante destacar que los movimientos con un alto grado de participación, tales como el movimiento verde, feminista, antirracista y otros tipos de movimientos no basados en la clase, han transitado de teorías basadas en los recursos de la movilización a teorías basadas en la autonomía cultural y la identidad colectiva (Buechler, 1993). Por ello, este trabajo pretende crear un modelo que permita identificar que las conductas solidarias de seguimiento de normas y proveer apoyo socio emocional durante el confinamiento pueden explicarse a través de compartir un destino común y una autocategorización dentro del grupo por medio de la identidad social que permitirían a los individuos enfrentar positivamente la pandemia.

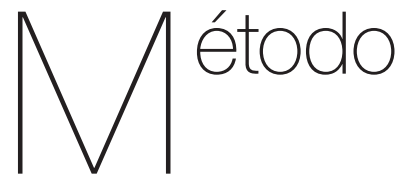

\section{Participantes}

La muestra incluyó participantes voluntarios que fueron contactados a través de redes sociales y en sus cuentas de correo electrónico. Se utilizó el formulario de Google Forms para recabar las respuestas. Participaron 1,000 hombres y mujeres del centro, norte y sur de la república mexicana (CDMX 477, Edo. Mex. 178, Hidalgo 97, Nuevo León 23, Jalisco 17, Guerrero 9, Tabasco 8, SLP 11, Querétaro 24, Puebla 17, Guanajuato 19, Baja California 20, Michoacán 9, Zacatecas 6, Morelos 14, Oaxaca 11, Veracruz 10, Yucatán 8, Chiapas 6, Quintana Roo 6, Durango 3, Nayarit 3, Tamaulipas 3, Aguascalientes 4, Sinaloa 6, Tlaxcala 3, Sonora 4, Campeche 1, Coahuila 1 y Chihuahua 2). La muestra fue accidental, invitándose a participar a todos aquellos que vivieran dentro del territorio nacional. Todos son usuarios con acceso a Internet de zonas urbanas. La Tabla 1 describe la edad, escolaridad y porcentaje de la muestra de participantes.

Tabla 1.

Datos sociodemográficos de la muestra

\begin{tabular}{llcc}
\hline & & N (\%) & M (DE) \\
\hline \multirow{2}{*}{ Escolaridad } & Edad & 1000 & $32.59(12.24)$ \\
& Primaria & $4(.4 \%)$ & \\
& Secundaria & $13(1.3 \%)$ & \\
& Preparatoria & $63(6.3 \%)$ & \\
\hline \multirow{4}{*}{ Técnica } & $32(3.2 \%)$ \\
& Licenciatura & $650(65.0 \%)$ \\
& Maestría & $123(12.3 \%)$ \\
& Doctorado & $115(11.5 \%)$ \\
\hline \multirow{2}{*}{ Sexo } & Hombre & $400(40 \%)$ \\
& Mujer & $600(60 \%)$ \\
\hline
\end{tabular}




\section{Instrumentos}

El instrumento fue adaptado a partir del Modelo de la Identidad Social elaborado en situaciones de emergencia y el estudio chileno de la emergencia derivada del sismo en 2010 (Drury, 2020; Drury, et al., 2016; Reicher, 2008). La investigación fue de autoreporte por lo que se siguió el establecimiento ético de la Universidad Nacional Autónoma de México de no pedir identificación alguna de los respondientes y no realizar preguntas que pudieran causar incomodidad o de naturaleza íntima. A continuación, se detallan las dimensiones, los reactivos, escala de respuesta y confiabilidad.

Del estudio de Drury et al. (2010), se recuperaron las siguientes dimensiones:

\section{Exposición a la Emergencia}

Sanitaria

Cuatro reactivos que hacen referencia al grado en el cuál las personas identifican que han sido expuestas a la pandemia. Ejemplo: 1. Siento que mi vida está en peligro, los tres primeros reactivos basados en el trabajo de Drury en el terremoto de Chile de 2010. En este estudio un cuarto reactivo fue redactado para dar cuenta del desabasto de víveres y medicamento derivados del confinamiento. 4. Me ha afectado directamente el desabasto de víveres y medicamentos por la pandemia. Las afirmaciones tienen un formato de respuesta dicotómico: cierto o falso. El cálculo de la dimensión se derivó al sumar los cuatro reactivos. La escala original de tres reactivos tuvo una confiabilidad $\alpha=.72$, mientras que la del estudio reportó un $\alpha$ de .53 .
Identidad Social con otros afectados por la emergencia sanitaria

Pieza central del estudio, esta dimensión refleja el grado de identificación con otras personas en el contexto del confinamiento derivado de la emergencia sanitaria. Ejemplo de los reactivos: 3. Me siento en una gran unidad con otros; 4. Siento que otras personas afectadas por la emergencia sanitaria son como yo. El formato de respuesta fue una escala de Likert que va de 1 "totalmente en desacuerdo" a 5 "Totalmente de acuerdo" (Drury et al., 2016). En el estudio original esta dimensión tuvo un $\alpha=.90$, y en este estudio se obtuvo un $\alpha=.70$.

\section{Observar Apoyo Social Emocional}

Conceptualmente, esta dimensión hace referencia a observar conductas de tipo emocional dando apoyo a otros. Se trata de un apoyo social y colectivo que refleja las tesis del aprendizaje social y la influencia en la ejecución de conductas a través de observar a otros ejecutándolas. Esta dimensión tiene tres reactivos, dos de ellos corresponden a la teoría de Drury et al. (2016): 1. Personas mostrando respeto por otros, 2. Personas mostrando preocupación por las necesidades de otros; y un último reactivo elaborado para este estudio: 3. Personas brindando apoyo emocional a otros. El formato de respuesta fue una escala de Likert que va de 1 "nunca" a 7 "muchas veces". El estudio de Drury et al. (2016) se realizó un análisis de correlación bivariado de Pearson $(=.84)$. Al tener un tercer factor, en este estudio se realizó el análisis de fiabilidad obteniendo un $\alpha=.84$. 


\section{Proveer Apoyo Social Emocional}

Esta dimensión hace referencia a la frecuencia con que las personas han brindado apoyo emocional a otros en el contexto inmediato al confinamiento. Consiste en tres reactivos, ejemplo: 1. He brindado apoyo emocional a otras personas. La dimensión corresponde al instrumento del estudio chileno (Drury et al., 2016). El formato de respuesta fue 1 "nunca" a 7 "muchas veces". La dimensión original reportó un $\alpha=.83$, en este estudio fue de .61.

\section{Destino Común}

Hace referencia al sentimiento de compartir el mismo destino ante la emergencia sanitaria. El formato de respuesta fue una escala de Likert que va de 1 "totalmente en desacuerdo" a 5 "Totalmente de acuerdo". La escala solo contiene dos reactivos: 1. Todos nos encontramos en peligro y 2 . Todos compartimos el mismo destino. La escala original del estudio de Drury et al., (2016) está compuesta por tres reactivos con un $\alpha=.77$, los dos reactivos que se conservaron para este estudio reportaron un $\alpha=.53)$.

Las siguientes dimensiones fueron creadas expresamente para este estudio:

\section{Observar Apoyo Social Coordinado} en Redes

En los estudios de acción colectiva el apoyo emocional en situaciones de emergencia obedecía a situaciones cara a cara, en cambio, en esta emergencia sanitaria, muchas de las conductas solidarias son visibles a través de grabaciones y transmitidas en redes sociales y programas de TV. Por ello, esta dimensión fue conceptualizada a través de tres reactivos, por ejemplo: 1. Las personas han llamado en redes sociales y otros medios a quedarse en casa y guardar sana distancia. El formato de respuesta fue una escala de Likert que va de 1 "totalmente en desacuerdo" a 5 "totalmente de acuerdo". La escala reporta un $\alpha=.70$.

\section{Observar Conductas Egoístas}

Se redactaron tres reactivos que hacen referencia a observar conductas egoístas, por ejemplo: 1. He observado a otras personas comportarse de forma egoísta. El formato de respuesta fue una escala de Likert que va de 1 "totalmente en desacuerdo" a 5 "totalmente de acuerdo". La escala reporta un $\alpha=.76$.

\section{Proveer Apoyo Emocional Coordinado en Redes}

Dada la importancia de proveer apoyo social como parte de las conductas solidarias o de apoyo entre el colectivo se redactaron una serie de reactivos que den cuenta del apoyo emocional brindado en Redes. Estos tres reactivos no se encuentran en el Instrumento original chileno, ejemplo de ellos: 3. Tengo amigos y familiares que sé que están al pendiente de mí a la distancia (por medio de Facebook o WhatsApp). El formato de respuesta fue una escala de Likert que va de 1 "totalmente en desacuerdo" a 5 "totalmente de acuerdo". La escala reporta un $\alpha=.64$.

\section{Seguimiento de Normas}

Ejemplo: 1. He tratado de seguir las indicaciones del personal de salud (confinamiento, sana distancia). Esta dimensión se apoya conceptualmente en las indicaciones de la Secretaría de Salud de México al iniciar la campaña de "Sana Distancia" (Salud, 2020). El formato de respuesta es 
una escala de Likert de siete puntos que va de 1 "nunca" a 7 "muchas veces". La escala reporta un alfa $=.804$.

\section{Hipótesis}

Relacionadas con la predicción de las conductas de solidaridad por la observación de conductas solidarias:

H1: la observación de conductas de apoyo social emocional predice la ejecución de conductas relacionadas a proveer apoyo social emocional, H2: proveer apoyo social emocional en redes, y H3: el seguimiento de normas.

H4: la observación de conductas de apoyo social coordinado en redes predice la ejecución de conductas relacionadas a proveer apoyo social emocional, H5: proveer apoyo social emocional en redes, y H6: el seguimiento de normas.

Relacionadas con la identidad social compartida:

H7: la identidad social compartida durante la emergencia sanitaria predice las conductas relacionadas a proveer apoyo social emocional, H8: el seguimiento de normas, y H9: proveer apoyo emocional en redes.

H10: el sentimiento de experimentar un destino común predice la identidad social compartida.

Relacionadas con la experiencia de la emergencia sanitaria:

H11: la exposición al desastre sanitario predice proveer apoyo social emocional, H12: proveer apoyo social coordinado en redes, H13: el seguimiento de normas, y H14: el destino común.

\section{Análisis de datos}

Para analizar los datos, se crearon modelos de medición que permitieran conocer la estructura factorial de cada dimensión a través del AFC. Un primer paso consistió en realizar estadísticos descriptivos y correlaciones entre las dimensiones. Posteriormente, se construyó un modelo de ecuación estructural que diera cuenta de las hipótesis relacionadas con la predicción de conductas relacionadas a proveer apoyo social emocional y al seguimiento de normas. Finalmente, se realizaron análisis de mediación que dieron cuenta de la identidad social compartida como un potente mediador de las conductas de solidaridad. Se tuvieron en cuenta los índices de ajuste propuestos por Hu y Bentler (1999), considerando un ajuste apropiado a los datos con un $\mathrm{CFI}=$ .95 , un $\mathrm{RMSA}=<.06$ y un $\mathrm{SRMR}=<.08$. Se utilizó el software $R$ versión 4.0.2 y la paquetería "lavaan" versión 0.6-7.

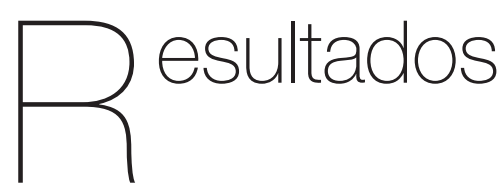

\section{Análisis descriptivos de cada dimensión}

Por medio del AFC se conceptualizaron 8 dimensiones que dan cuenta del Modelo de Identidad Social. Aunado a ello, se reporta la media y desviación estándar de cada dimensión en la Tabla 2. 
Tabla 2.

Media y desviación estándar de cada dimensión del Modelo

\begin{tabular}{lccc}
\hline \multicolumn{1}{c}{ Dimensión } & Mínimo & Máximo & M (DE) \\
\hline $\begin{array}{l}\text { Exposición a la } \\
\text { emergencia sanitaria }\end{array}$ & 1 & 4 & $1.99(1.15)$ \\
$\begin{array}{l}\text { Destino Común } \\
\text { Identidad Social }\end{array}$ & 1 & 5 & $3.39(1.10)$ \\
$\begin{array}{l}\text { Proveer Apoyo } \\
\text { Social Emocional }\end{array}$ & 1 & 7 & $5.98(.885)$ \\
$\begin{array}{l}\text { Proveer Apoyo Emocional } \\
\text { Coordinado en Redes }\end{array}$ & 1 & 5 & $4.41(.725)$ \\
$\begin{array}{l}\text { Seguimiento Normas } \\
\text { Observar Apoyo }\end{array}$ & 1 & 5 & $4.64(.583)$ \\
$\begin{array}{l}\text { Social Emocional } \\
\text { Observar Apoyo Social } \\
\text { Coordinado en Redes }\end{array}$ & 1 & 7 & $4.50(1.33)$ \\
$\begin{array}{l}\text { Observar } \\
\text { Conductas Egoístas }\end{array}$ & 1 & 5 & $4.07(.852)$ \\
\hline
\end{tabular}

Nota: ${ }^{*} p<.05,{ }^{* *} p<.01,{ }^{* * *} p<.001$, elaboración propia

\section{Análisis Factorial Confirmatorio de las dimensiones con respuesta ordenada}

Se realizó un AFC para las dimensiones que planea el estudio con tipo de respuesta de escala Likert. La dimensión relacionada con la exposición a la emergencia sanitaria fue dejada de lado dado que se incluirá en el modelo completo de ecuación estructural como una variable observada.

Los resultados estadísticos indican un ajuste adecuado a los datos $\chi 2$ (247) $=732.53 \mathrm{p}<.001, \mathrm{CFI}=.96$; RMSEA = $.045 ; \mathrm{SRMR}=.049$ (Hu \& Bentler, 1999). La Figura 1 muestra las cargas factoriales superiores a .40 en todas las dimensiones, así como las correlaciones entre dimensiones.

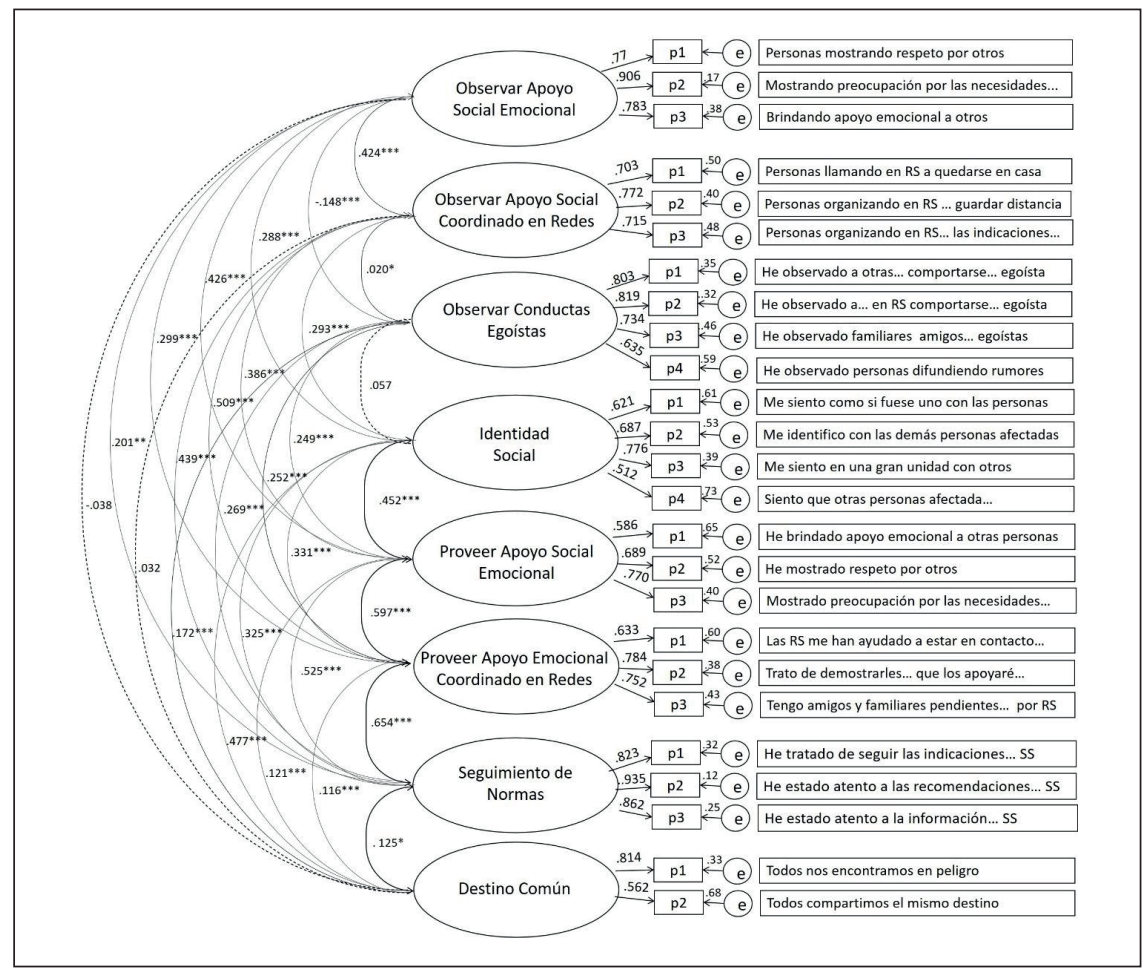

Figura 1. Análisis Factorial Confirmatorio de las variables latentes, elaboración propia 


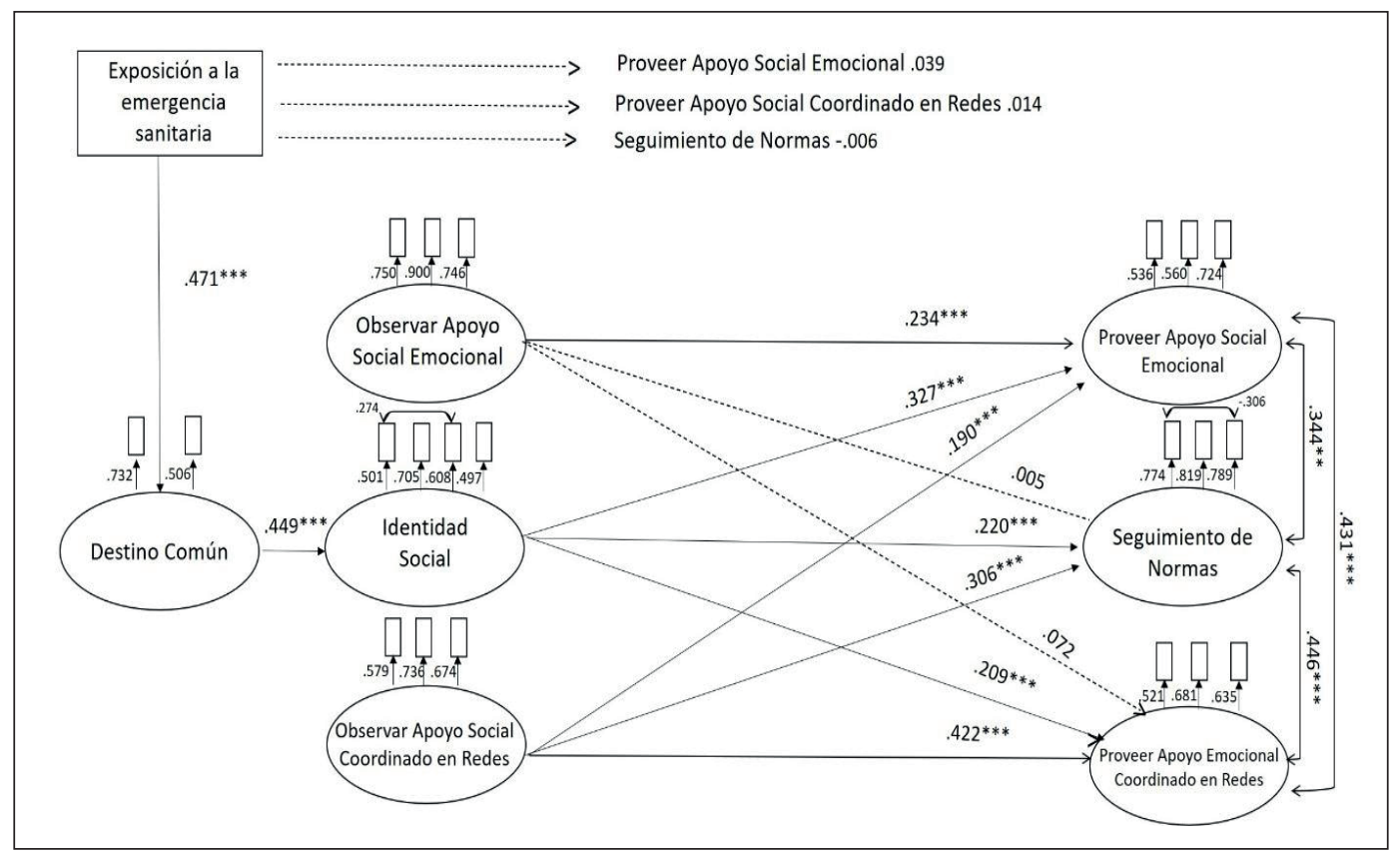

Figura 2. Ecuación Estructural del Modelo Elaborado de la Identidad Social aplicado a la solidaridad ante la emergencia sanitaria COVID-19

\section{Modelo Elaborado de la Identidad Social}

El modelo tuvo un ajuste adecuado, aunque se retiró del modelo la dimensión de observar conductas egoístas ya que mostró poca relación con las demás dimensiones propuestas. El ajuste del modelo teórico fue adecuado $\chi 2(190)$ $=589.46 \mathrm{p}<.001, \mathrm{CFI}=.93 ; \mathrm{RMSEA}=$ $.046 ; \mathrm{SRMR}=.060$ (Hu \& Bentler, 1999).

Predicción de las conductas de solidaridad por la observación de conductas solidarias

Los datos indicaron un apoyo a la $\mathrm{H} 1$, la observación de conductas de apoyo social emocional predice la ejecución de conductas relacionadas a proveer apoyo social emocional $(\beta=.23, p>$.001). Sin embargo, el observar conductas de apoyo social emocional no predice el proveer apoyo emocional coordinado en redes $\mathrm{H} 2$ ( $\beta=.07, p>.050)$, ni el seguimiento de normas $(\beta=.00, p>$.050). En cambio, el observar apoyo social coordinado en redes predice el proveer apoyo emocional coordinado en redes $\mathrm{H} 5$ ( $\beta=.42, \mathrm{p}<$ .001), el seguimiento de normas H6 ( $\beta=$ $.30, \mathrm{p}<.001)$ y $\mathrm{H} 4$ proveer apoyo social emocional $(\beta=.19, p<.001)$.

Predicción de las conductas de solidaridad por la identidad social

La identificación social con otras personas durante la emergencia sanitaria predice el proveer apoyo social emocional $H 7$ ( $\beta=.32, p<.001)$, el seguimiento de normas $\mathrm{H} 8(\beta=.22, p<.001)$ y proveer apoyo social emocional en redes H9 ( $\beta=.20, p<.001)$. Mientras que el experimentar un destino común impacta sobre la identificación social $\mathrm{H} 10(\beta=$ $.53, p<.001)$. 


\section{Exposición a la emergencia sanitaria}

Por sí misma, la exposición a la emergencia sanitaria no predice el proveer apoyo social emocional $\mathrm{H} 11$ ( $\beta=.03$, $\mathrm{p}$ $<$.050), proveer apoyo social coordinado en redes $\mathrm{H} 12$ ( $\beta=.01, p<.050)$, ni el seguimiento de normas H13 $(\beta=-.00, p<$ .050). En cambio, predice el experimentar un destino común H14 ( $\beta=.47, p<.001)$. Finalmente, el destino común predice la identidad social $(\beta=.44, p<.001)$.

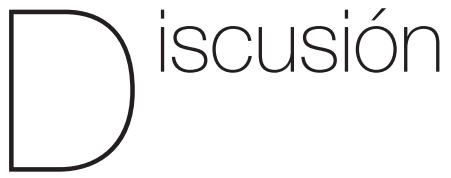

Existen dos resultados clave de la presente investigación: primero, se testan las hipótesis referentes a la predicción de las conductas solidarias a través de la observación y, segundo, se testa la importancia de la predicción de conductas solidarias a través de la identidad social compartida, derivada de la existencia de un sentimiento de compartir un destino común ante la exposición a la emergencia sanitaria.

Existe evidencia de que las personas ejecutamos conductas por el simple hecho de observarlas (Bandura, 1982; Drury et al., 2016). A diferencia del aprendizaje por medio del conocimiento, el aprendizaje social basa la conducta de aprendizaje en observar y modelar (Bandura, 1982). Con base en estos principios, se hipotetizó (hipótesis 1 a la 6) que observar conductas solidarias de tipo emocional (observé personas mostrando respeto por otros) y de apoyo social coordinado en redes (personas llamando en redes a quedarse en casa) predecía la ejecución de conductas solidarias tales como seguir normas, proveer apoyo emocional coordinado en redes y proveer apoyo social emocional a otras personas. Los resultados mostraron que, en efecto, observar conductas de apoyo social emocional predice el proveerlas (.24), sin embargo, no predice el seguimiento de normas sanitarias, ni el proveer apoyo emocional coordinado a través de las redes sociales virtuales. Un mayor efecto tuvo el observar apoyo social coordinado en redes, es decir, observar en las redes a personas organizándose para guardar sana distancia o llamando a quedarse en casa, que predice el seguimiento de normas sanitarias (.30), proveer apoyo social emocional al mostrar respeto y preocupación por otros (.19) y proveer apoyo emocional coordinado en redes al estar en contacto, demostrar apoyo y estar al pendiente de otros a través de las redes sociales (.42). Estos resultados concuerdan con la literatura clásica que enfatiza la observación de conductas solidarias ante una emergencia como predictores de ejecución de conductas solidarias (Cocking et al., 2009; Drury et al., 2016).

Con ello, se evidencia la importancia de observar conductas solidarias en redes sociales, dado que el modelo original, planteado durante el sismo chileno de 2010, implicaba la observación directa de estas acciones y la actual imposibilidad de observación directa durante este confinamiento; este planteamiento ubica la observación en redes sociales. Debido al confinamiento se ha observado que la vida en redes sociales ha incrementado sustancialmente (Pérez-Escoda et al., 2020). Tan es así que los individuos se han vertido a escuchar noticias de manera directa en las redes sociales en 
comparación con los medios tradicionales de radio y televisión (Allington et al., 2020; UNAM, 2020). Estos resultados son relevantes si se toma en toma en cuenta que una mayor cantidad de apoyo social coordinado en redes predice no sólo el proveer apoyo emocional coordinado en redes sino también el seguimiento de normas sanitarias. Sin embargo, las redes sociales tienen dos sentidos contrastantes: por un lado, la posibilidad de contacto y observación de conductas solidarias y, por el otro lado, son fuente de difusión de rumores, campañas de odio, información falsa y teorías conspiracionistas. Este lado "oscuro" de la red aleja a los individuos de conductas de solidaridad con otros ciudadanos. Tan es así que se ha encontrado que una mayor aceptación de creencias conspiracionistas sobre el COVID-19 se relacionan a un menor seguimiento de conductas protectoras de la salud para enfrentar el contagio y a un mayor uso de redes sociales para obtener información sobre la pandemia.

Las hipótesis relacionadas con la identidad social evidencian una predicción alta de las conductas de solidaridad. Esto es aún más relevante en el caso de proveer apoyo social emocional siendo la identidad social su mayor predictor (.33), en comparación con observar el apoyo social emocional (.23) y observar el apoyo emocional en redes (.19). Este patrón de resultados es consistente con la literatura previa y los estudios de Drury (Cocking et al., 2009; Drury et al., 2016) sobre la identidad social y su efecto sobre la conducta solidaria en emergencia. De hecho, la sola exposición a la emergencia sanitaria no predice las conductas de solidaridad (proveer apoyo social emocional .04, proveer apoyo social coordinado en redes .01 y seguimiento de normas -.01).
Es necesaria la identificación social para la elicitación de las conductas solidarias. Esta información concuerda con las tesis de que la exposición a la emergencia sanitaria predice el sentimiento de destino común (.47) y éste, la identidad social compartida con otros participantes, siendo esta última la mejor predictora para las conductas de solidaridad. Contrario al planteamiento tradicional acerca de la conducta de masa como irracional y elicitadora de conductas agresivas (Reicher, 2008), lo cierto es que la conducta colectiva es racional y facilitadora de solidaridad ante emergencias de corte sanitario. Con ello, se corrobora también que la acción colectiva es posible (Reicher, 2008). Esta acción va del empoderamiento colectivo y la solidaridad a través de la identificación colectiva - que va más allá de la identidad individual - manifestando así, que los colectivos tienen mucho que decir (Buechler, 1993; Melucci, 1989; Sudbury, 2012) y mucho que hacer.

Tan es así que la autocategorización como parte de un colectivo que enfrenta una situación de emergencia sanitaria nos llevaría a compartir la identidad (Turner et al., 1994), a influenciar la ejecución de conductas solidarias y, con ello, tener una actitud más crítica (Fantasia, 1989) y una autoconcepción más clara de la situación que se vive. Promover el sentimiento de destino común compartido entre los ciudadanos, así como la observación de conductas de apoyo emocional y social en redes puede llevar a un mayor seguimiento de conductas de salud para evitar el contagio y, con ello, alejarnos de las tesis tradicionales que identifican a la masa como irracional y egoísta. De esta manera, podríamos enfrentar, de una manera más clara, el seguimiento de normas sanitarias ante el avance de la pandemia 
y otras situaciones de emergencia en nuestro país.

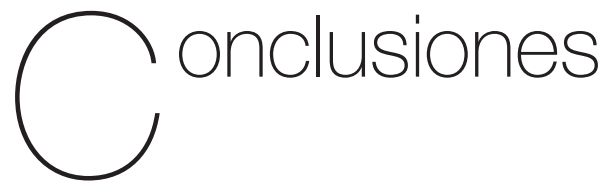

Ciertas limitaciones en este estudio pueden ser señaladas para guiar futuras investigaciones. Por ejemplo, la limitación a una muestra derivada de contactos en el interior de la república mexicana, investigaciones futuras deben ampliar el campo de estudio a otras poblaciones. La teoría de la identidad social comienza a ser una de las teorías más extendidas en la explicación acerca de la ejecución de conductas solidarias, empoderamiento en movimientos sociales y servicios de emergencia entre otros, por lo que la investigación en México potencialmente podría cubrir otras temáticas relevantes (Buechler, 1993; Mawson, 2005). A pesar de esto, el presente trabajo constituye uno de los primeros intentos en conjuntar la teoría de la identidad social compartida en situaciones de emergencia en México. Además, la presente investigación contribuye al creciente cuerpo de investigación que sugiere que la identidad social es una pieza clave para la sobrevivencia en situaciones de emergencia.

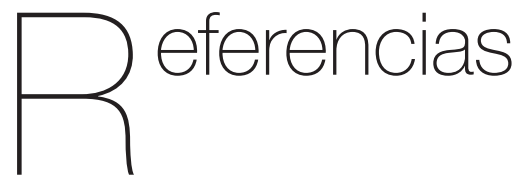

Allington, D., Duffy, B., Wessely, S., Dhavan, N., \& Rubin, J. (2020). Health-protective behaviour, social media usage, and conspiracy belief during the COVID-19 public health emergency. Psychological Medicine, 51(10), 1763-1769. https://doi. org/10.1017/S003329172000224X

Bandura, A. (1982). Teoría del Aprendizaje Social. Madrid: Espasa Calpe.

Brooks, S. K., Webster, R. K., Smith, L. E., Woodland, L., Wessely, S., Greenberg, N., \& Rubin, G. J. (2020). The psychological impact of quarantine and how to reduce it: rapid review of the evidence. The Lancet, 395(10227), 912-920. https://doi. org/10.1016/S0140-6736(20)30460-8

Buechler, S. M. (1993). Beyond resource mobilization? Emerging Trends in Social Movement Theory. Sociological Quarterly, 34(2), 217-235. https://doi. org/10.1111/j.1533-8525.1993.tb00388.x

Cocking, C., Drury, J., \& Reicher, S. (2009). The psychology of crowd behaviour in emergency evacuations: Results from two interview studies and implications for the Fire and Rescue Services. Irish Journal of Psychology, 30(1-2), 59-73. https://doi.org/ 10.1080/03033910.2009.10446298

Drury, J. (2018). The role of social identity processes in mass emergency behaviour: An integrative review. European Review of Social Psychology, 29(1), 38-81. https://doi. org/10.1080/10463283.2018.1471948

Drury, J. (2020). Recent developments in the psychology of crowds and collective behaviour. Current Opinion in Psychology, 35, 12-16. https://doi.org/10.1016/j. copsyc.2020.02.005

Drury, J., Brown, R., González, R., \& Miranda, D. (2016). Emergent social identity and observing social support predict social support provided by survivors in a disaster: 
Solidarity in the 2010 Chile earthquake. European Journal of Social Psychology, 46(2), 209-223. https://doi.org/10.1002/ ejsp.2146

Drury, J., Cocking, C., \& Reicher, S. (2009a). Everyone for themselves? A comparative study of crowd solidarity among emergency survivors. British Journal of Social Psychology, 48(3), 487-506. https:// doi.org/10.1348/014466608X357893

Drury, J., Cocking, C., \& Reicher, S. (2009b). The Nature of Collective Resilience: Survivor Reactions to the 2005 London Bombings. International Journal of Mass Emergencies and Disasters, 27(1), 66-95.

Drury, J., \& Reicher, S. (1999). The Intergroup Dynamics of Collective Empowerment: Substantiating the Social Identity Model of Crowd Behavior. Group Processes and Intergroup Relations, 2(4), 381-402. https:// doi.org/10.1177/1368430299024005

Fantasia, R. (1989). Cultures of Solidarity: Consciousness, Action, and Contemporary American Workers. Rick Fantasia. London England, University of California Press, Ltd. https://doi.org/10.1086/229211

Featherstone, M. (1995). Undoing culture: globalization, postmodernism and identity (S. Publications, Ed.). London, Thousand Oaks,.

Fernández-Poncela, A. M. (2012). Psicología de masas, identidad social, epidemias y rumores: la influenza en...: Discovery Service para Universidad Peruana Unión. Sociológica, 27(76), 189-230. Retrieved from http:// eds.a.ebscohost.com/eds/pdfviewer/ pdfviewer?vid=0\&sid=75ad6a4c- b $588-4$ b 0 e - b b 35 c87ffb0c2e85\%40sessionmgr4006

Hu, L. T., \& Bentler, P. M. (1999). Cutoff criteria for fit indexes in covariance structure analysis: Conventional criteria versus new alternatives. Structural Equation Modeling, 6(1), 1-55. https://doi. org/10.1080/10705519909540118

Mawson, A. R. (2005). Understanding mass panic and other collective responses to threat and disaster. Psychiatry, 68(2), 95-113. https://doi.org/10.1521/ psyc.2005.68.2.95

Melucci, A. (1989). Nomads of the Present: Social Movements and Individual Needs in Contemporary Society. London: H. Radius, Ed.

Pérez-Escoda, A., Jiménez-Narros, C., Perlado-Lamo-de-espinosa, M., \& Pedrero-Esteban, L. M. (2020). Social networks' engagement during the COVID-19 pandemic in Spain: Health media vs. healthcare professionals. International Journal of Environmental Research and Public Health, 17(14), 1-17. https://doi. org/10.3390/ijerph17145261

Redacción. (2020). Infodemia masiva invade sociedades de México y el mundo. Retrieved from 08/08/2020 website: https:// www.eleconomista.com.mx/arteseideas/ Infodemia-masiva-invade-sociedades-deMexico-y-el-mundo-20200805-0064.html

Reicher, S. (2008). The Psychology of Crowd Dynamics. In M. A. Hogg \& R. S. Tintale (Eds.), Blackwell Handbook of Social Psychology: Group Processes (pp. 182208). Oxford: Oxford: Blackwell. https://doi. org/10.1002/9780470998458.ch8 
Sandín, B., Valiente, R. M., García-Escalera, J., \& Chorot, P. (2020). Impacto psicológico de la pandemia de COVID-19: Efectos negativos y positivos en población española asociados al periodo de confinamiento nacional. Revista de Psicopatología y Psicología Clínica, 25(1), 1-22. https://doi. org/10.5944/rppc.27569

Solnit, R. (2009). A paradise built in hell: The extraordinary communities that arise in disaster. New York: Viking.

Sudbury, J. (2012). Ethnic and Racial Studies ( $R e$ ) constructing multiracial blackness : women's activism, difference and collective identity in Britain. Ethnic and Racial Studies, 24(1), 29-49. https://doi. org/10.1080/0141987002000653

Turner, J. C., Oakes, P. J., Haslam, S. A., \& McGarty, C. (1994). Self and Collective: Cognition and Social Context. Personality and Social Psychology Bulletin, 20(5), 454-463. https://doi. org/10.1177/0146167294205002
Uluğ, Ö. M., \& Acar, Y. G. (2019). 'Names will never hurt us': A qualitative exploration of çapulcu identity through the eyes of Gezi Park protesters. British Journal of Social Psychology, 58(3), 714-729. https://doi. org/10.1111/bjso.12305

UNAM. (2020). Riesgos y vulnerabilidades por COVID-19 reestructuran al mundo. Retrieved from https://www.dgcs.unam.mx/boletin/bdboletin/2020_698.html?fbclid=IwA R20DBCaMKnIBhimcYfBKfug4PFZIQNMAQ6PxhEoC3bKDRq8XZGshOElois

Zandifar, A., \& Badrfam, R. (2020). Iranian mental health during the COVID-19 epidemic. Asian Journal of Psychiatry, 51(February), $101990 . \quad$ https://doi. org/10.1016/j.ajp.2020.101990

Zeitz, K. M., Tan, H. M., Grief, M., Couns, P. C., \& Zeitz, C. J. (2009). Crowd behavior at mass gatherings: A literature review. Prehospital and Disaster Medicine, 24(1), 32-38. https:// doi.org/10.1017/S1049023X00006518 